Bull. Korean Math. Soc. 50 (2013), No. 2, pp. 559-581

http://dx.doi.org/10.4134/BKMS.2013.50.2.559

\title{
ON SOME RESULTS OF BUMP-CHOIE AND CHOIE-KIM
}

\author{
Joseph HundLey
}

\begin{abstract}
This paper is motivated by a 2001 paper of Choie and Kim and a 2006 paper of Bump and Choie. The paper of Choie and Kim extends an earlier result of Bol for elliptic modular forms to the setting of Siegel and Jacobi forms. The paper of Bump and Choie provides a representation theoretic interpretation of the phenomenon, and shows how a natural generalization of Choie and Kim's result on Siegel modular forms follows from a natural conjecture regarding $(g, K)$-modules. In this paper, it is shown that the conjecture of Bump and Choie follows from work of Boe. A second proof which is along the lines of the proof given by Bump and Choie in the genus 2 case is also included, as is a similar treatment of the result of Choie and Kim on Jacobi forms.
\end{abstract}

\section{Introduction}

The purpose of this note is to give a representation-theoretic interpretation for the results of Choie and Kim [7], continuing a program begun by Bump and Choie in [6]. The motivation for [7] is to generalize a certain result of Bol [4] from elliptic modular forms to Siegel and Jacobi forms. An interesting consequence is that the $(r+1) \underline{\text { st }}$ derivative of a meromorphic modular form of weight $-r$ is a meromorphic modular form of weight $r+2$. This is striking because no other derivative of the same modular form will be modular. More precisely, each derivative is expressed as a linear combinations of forms of various weights, only the $(r+1)$ st derivative has the property that the contributions from all weights save one are zero. As explained in [6], it is possible to consider instead a slightly different differential operator (the Maass raising operator)

Received November 28, 2011.

2010 Mathematics Subject Classification. 11F70, 11F46, 11F50.

Key words and phrases. Siegel modular forms, Jacobi forms, meromorphic automorphic forms, $(g, K)$-modules, generalized Verma modules, Bol's result.

Most of this research was completed in 2006-07 while the author was a post-doc at Pohang University of Science and Technology and then at Tel Aviv University. He would like to thank both institutions for their hospitality, as well as his hosts, Professor YoungJu Choie and Professor David Ginzburg, respectively. He would also like to thank Professor Choie for suggesting the problem and for many useful discussions. This paper was written while the author was supported by NSF Grant DMS-1001792. 
such that all derivatives are automorphic forms, but none save the $(r+1) \underline{\text { st }}$ is meromorphic.

The results of [7] extend Bol's result to Siegel modular forms as well as Jacobi forms, subject to certain restrictions. For the Siegel case, one considers a meromorphic Siegel modular form of genus $n$ and weight $-r+\frac{n-1}{2}$. The usual derivative $\frac{d}{d z}$ of a modular form in the upper half plane is replaced by the "determinant differential operator" in the Siegel case. Just as before, one finds that the $(r+1)$ st iterate of this operator, yields a meromorphic Siegel or Jacobi form of weight $r+2+\frac{n-1}{2}$. Further one does not get a meromorphic modular form for any derivative other than the $(r+1)^{\text {st }}$. The Jacobi case is similar. Let us refer to this as "recovery at $r+1$ ".

The Siegel case is considered from another perspective in [6], and the important task of translating the phenomenon into a representation-theoretic context is carried out. It is explained how certain vectors in $(\mathfrak{s p}(2 n, \mathbb{C}), \widetilde{U}(n))$-modules generalize meromorphic modular forms of negative weight, and how a conjecture (Conjecture 2 of [6]) would explain and also generalize the natural extension (Conjecture 1 of [6]) of recovery at $r+1$.

The goals of this paper are as follows: first, to prove Conjecture 2 of [6]; second, to generalize Theorem 3 of [6]; third, to give a similar treatment of the Jacobi case of [7].

Two proofs of Conjecture 2 of [6] are given. The first, short proof, is based on deducing the conjecture from general results of [2], which apply to any semisimple complex Lie algebra. Since the main program is to make the representationtheoretic explanations for the "recovery" behavior of Siegel and Jacobi modular forms as transparent as possible, a detailed proof for the case $\mathfrak{s p}(2 n, \mathbb{C})$ is included. A second, longer, proof, along the lines of that given in [6] for the case $n=2$ is then presented.

The generalization of Theorem 3 of [6] is given in Section 5. It helps to explain why, regardless of the the genus $n$, one is able to prove Conjecture 2 using only the Laplace-Beltrami operator, without considering any other elements of the center of the universal enveloping algebra.

In Section 6, notation for the Jacobi case is introduced. The main theorem in the Jacobi case, Theorem 7.2, is then given in Section 7. It generalizes Corollary 3.6 of [7]. The proof is based on extending Jacobi forms to functions on a large Siegel upper half-space and applying results from the Siegel case. In Section 8 , Theorem 8.7, which may be regarded as a Jacobi version of Conjecture 2 is stated and proved. In principle, this result could be the basis for a second proof of Theorem 7.2, but it's not entirely trivial: like Conjecture 2 of [6], Theorem 8.7 deals with certain elements of the universal enveloping algebra which correspond to Maass's raising operators. The relationship between these raising operators and the heat operator and determinant differential operator considered in [7] is somewhat indirect. In the Siegel case, the relationship is given in Lemma 7.3, which relies on results of Harris and Maass. In order 
to deduce Theorem 7.2 from Theorem 8.7, some Jacobi version of this lemma would be required. Even though no second proof of Theorem 7.2 is obtained, it seems that Theorem 8.7, and particularly its proof, may be of sufficient independent interest to warrant inclusion. The proof is based on embedding a smaller symplectic Lie algebra in the universal enveloping algebra of the Jacobi Lie algebra in a nonstandard way, and applying results from the Siegel case to this subalgebra.

\section{Reduction}

As mentioned above, this paper attempts to continue a program begun in [6]. Accordingly, we make free use of notation and terminology introduced in [6], and generally begin not at the beginning, but where [6] leaves off.

To briefly recall the statement of Conjecture 2 , recall that a $\left(\mathfrak{g}_{\mathbb{C}}, \widetilde{K}\right)$-module is a vector space equipped with actions of $\mathfrak{g}_{\mathbb{C}}:=\mathfrak{s p}(2 n, \mathbb{C})$ and of $\widetilde{K}=\widetilde{U}(n)$, subject to certain compatibility conditions described on [6], pp. 116-117. Here $\widetilde{U}(n)$ is the preimage of the unitary group $U(n) \subset S p(2 n, \mathbb{R})$ in the metaplectic double cover $\widetilde{S p}(2 n, \mathbb{R})$ of $S p(2 n, \mathbb{R})$.

Let $(\pi, V)$ be such an object. A vector $v \in V$ is said to be semispherical if the action of $\widetilde{K}$ preserves the one dimensional subspace $v$ spans. In this case $\widetilde{K}$ acts on $v$ by a character. Following [6], p. 121, we denote this character by $\operatorname{det}^{k}$, with $k$ being a half integer. The vector $v$ is said to be holomorphic if it is semispherical and, in addition, annihilated by certain differential operators $\pi\left(L_{X}\right)$ described on [6], p. 120. Rather than the determinant differential operator, one works with a different, but closely related differential operator, namely the Maass raising operator $M_{+}$defined on p. 123 of [6]. Conjecture 2 of [6] then states that if $v$ is a holomorphic vector of weight $-r+\frac{n-1}{2}$, then $M_{+}^{r+1} v$ is holomorphic of weight $r+2+\frac{n-1}{2}$.

In order to prove this, it is useful to simply forget the $\widetilde{K}$-module structure, and regard $V$ as a module over the ring $U\left(\mathfrak{g}_{\mathbb{C}}\right)$. Let $\mathfrak{k}$ denote the Lie algebra of $\widetilde{K}$. Then the compatibility conditions of a $\left(\mathfrak{g}_{\mathbb{C}}, \widetilde{K}\right)$-module force $\mathfrak{k}$ to act on the span of $v$ by a character, $\pi(X) v=k \operatorname{Tr}(X) v$, where $k \in \frac{1}{2} \mathbb{Z}$ is the weight, and $\operatorname{Tr}(X)$ denotes the trace of $X \in \mathfrak{k}$, regarded as an element of $\mathfrak{g l}(n, \mathbb{C})$, as opposed to $\mathfrak{s p}(2 n, \mathbb{R})$. To be precise, let $c$ be the Cayley transform, as on [6], p. 120. Then for $X \in \mathfrak{k}$, the matrix $c X c^{-1}$ is of the form $\left({ }^{X_{0}} \bar{X}_{0}\right)$, with $X_{0} \in \mathfrak{s u}(n)$ and $\operatorname{Tr}(X)$ denotes the trace of $X_{0}$. Note that $\bar{X}_{0}=-{ }^{\top} X_{0}$, for $X_{0} \in \mathfrak{s u}(n)$. It follows by linearity that the vector $v_{0}:=\pi(c) v$ satisfies

(Weight $k$ )

$$
\pi\left(\begin{array}{cc}
X & \\
& -{ }^{\top} X
\end{array}\right) v_{0}=k \operatorname{Tr}(X) v_{0} \quad \forall X \in \mathfrak{g l}(n, \mathbb{C}) .
$$

Moreover, by holomorphicity, (cf. the definition of $L_{X}$ on [6], p. 120)

$(\mathrm{Hol})$

$$
\pi\left(\begin{array}{cc}
0 & 0 \\
X & 0
\end{array}\right) v_{0}=0, \quad \forall X \in \operatorname{Mat}_{n}(\mathbb{C}), \text { symmetric. }
$$


Moreover, if $V$ is a $\left(\mathfrak{g}_{\mathbb{C}}, \widetilde{K}\right)$-module, and $v_{0} \in V$ satisfies (Weight $k$ ), then it follows from the compatibility conditions for a $\left(\mathfrak{g}_{\mathbb{C}}, \widetilde{K}\right)$-module that $v_{0}$ is semispherical of weight $k$. Hence, Conjecture 2 of [6] reduces to the following proposition.

Proposition 2.1. Let $V$ be a $U\left(\mathfrak{g}_{\mathbb{C}}\right)$ module, and suppose that $V$ contains a vector $v_{0}$ satisfying $(\mathrm{Hol})$ and $\left(\right.$ Weight $\left.-r+\frac{n-1}{2}\right)$. Then $\hat{M}_{+}^{r+1} v_{0}$ satisfies $(\mathrm{Hol})$ and (Weight $r+2+\frac{n-1}{2}$ ). Moreover, if $k \neq r+1$, then $\hat{M}_{+}^{k} v_{0}$ does not satisfy (Hol), where $\hat{M}_{+}=\operatorname{det} \hat{R}_{X_{i j}}$, with the same convention as on $p .123$ of $[6]$.

\section{Short proof of Proposition 2.1}

One may give a short proof of Proposition 2.1 using a modest refinement of Theorem 4.4 of [2]. Thus, take $\mathfrak{g}$ now to be a finite dimensional complex semisimple Lie algebra as in [2], and let $\mathfrak{p}$ be a Hermitian symmetric parabolic subalgebra. Write $\mathfrak{p}=\mathfrak{u}^{+} \oplus \mathfrak{r}$ where $\mathfrak{u}^{+}$is the unipotent radical of $\mathfrak{p}$ and $\mathfrak{r}$ is reductive, and write $\mathfrak{r}=\mathfrak{g}_{S}+\mathfrak{h}$, where $\mathfrak{g}_{S}$ is the derived subalgebra and $\mathfrak{h}$ is a Cartan subalgebra. Let $\lambda: \mathfrak{h} \rightarrow \mathbb{C}$ be a linear mapping which is trivial on $\mathfrak{h} \cap \mathfrak{g}_{S}$, and let pr be the natural projection $\mathfrak{p} \rightarrow \mathfrak{h} /\left(\mathfrak{h} \cap \mathfrak{g}_{S}\right)$. One considers those vectors $v$ in a $U(\mathfrak{g})$-module $V$ which satisfy the following natural generalization of (Hol) and (Weight $k$ ):

(Hol wt $\lambda) \quad X v=\lambda(\operatorname{pr}(X)) v \quad(\forall X \in \mathfrak{p})$.

Then one has, from [2], a necessary and sufficient condition for a representation which is generated by a vector which satisfies (Hol wt $\lambda$ ) to contain a vector which satisfies (Hol wt $\lambda^{\prime}$ ), where $\lambda^{\prime} \neq \lambda$. In order to state this condition, one needs a bit more notation. First, let $\mathfrak{u}^{-}$denote the nilpotent radical of the parabolic subalgebra opposite $\mathfrak{p}$, so that $\mathfrak{g}=\mathfrak{p} \oplus \mathfrak{u}^{-}$, and let $\rho^{S}$ equal half the sum of the roots of $\mathfrak{h}$ in $\mathfrak{u}^{+}$. Next, let $\alpha$ denote the unique noncompact simple root of $\mathfrak{h}$, let $\mathfrak{g}_{\alpha}$ be the $\alpha$-eigenspace of $\mathfrak{h}$ in $\mathfrak{g}$, let $h_{\alpha}$ denote the unique element of $\left[\mathfrak{g}_{\alpha}, \mathfrak{g}_{\alpha}\right]$ with $\alpha\left(h_{\alpha}\right)=2$. Then the space $\mathfrak{h} /\left(\mathfrak{h} \cap \mathfrak{g}_{S}\right)$ is one dimensional and spanned by the image of $h_{\alpha}$. Let $\omega_{\alpha}: \mathfrak{h} \rightarrow \mathbb{C}$ be the unique element which is trivial on $\left(\mathfrak{h} \cap \mathfrak{g}_{S}\right)$ and sends $h_{\alpha}$ to 1 .

Theorem 3.1. Keep the notation of the previous paragraph and let $\lambda, \lambda^{\prime}$ : $\mathfrak{h} /\left(\mathfrak{h} \cap \mathfrak{g}_{S}\right) \rightarrow \mathbb{C}$ be two linear maps. The following are equivalent.

(1) There exists a $U(\mathfrak{g})$-module $V$, which is generated by a vector $e$ which satisfies (Hol wt $\lambda$ ) and contains a second vector $e^{\prime}$ satisfying (Hol wt $\left.\lambda^{\prime}\right)$,

(2) There is a nontrivial $U(\mathfrak{g})$-module map from the scalar generalized Verma module $U(\mathfrak{g}) \otimes_{U(\mathfrak{p})} \mathbb{C} e_{\lambda^{\prime}}$ to the scalar generalized Verma module $U(\mathfrak{g}) \otimes_{U(\mathfrak{p})} \mathbb{C} e_{\lambda}$,

(3) The algebra of $\mathfrak{g}_{S}$-invariants in $U\left(\mathfrak{u}^{-}\right)$is of the form $\mathbb{C}\left[u_{r}\right]$, and there is a nonnegative integer $k$ such that

$$
\lambda=\left(k-\rho^{S}\left(h_{\alpha}\right)\right) \omega_{\alpha}, \quad \lambda^{\prime}=\left(-k-\rho^{S}\left(h_{\alpha}\right)\right) \omega_{\alpha} .
$$


Moreover, when the three equivalent conditions hold, the vector $e^{\prime}$ mentioned in (1) is a scalar multiple of $u_{r}^{k} . e$, and the map mentioned in (2) sends $1 \otimes e_{\lambda^{\prime}}$ to a scalar multiple of $u_{k}^{r} \otimes e_{\lambda}$ (Note that this map is completely determined by its value on $\left.1 \otimes e_{\lambda^{\prime}}\right)$.

Sketch of proof. The statement given above goes slightly beyond what is stated in [2], but the arguments in [2] prove it. Indeed, it is clear that $(2) \Longrightarrow(1)$, for if $\phi: U(\mathfrak{g}) \otimes_{U(\mathfrak{p})} \mathbb{C} e_{\lambda^{\prime}} \rightarrow U(\mathfrak{g}) \otimes_{U(\mathfrak{p})} \mathbb{C} e_{\lambda}$ is any nonzero map, then (1) is satisfied with $V=U(\mathfrak{g}) \otimes_{U(\mathfrak{p})} \mathbb{C} e_{\lambda}, e=e_{\lambda}$ and $e^{\prime}=\phi\left(e_{\lambda^{\prime}}\right)$. Further, if $V$ is any $U(\mathfrak{g})$-module generated by a vector $e$ which satisfies (Hol wt $\lambda$ ), then $V=U\left(\mathfrak{u}^{-}\right) e$. Arguing as on p. 796 of [2], if $u \in U\left(\mathfrak{u}^{-}\right)$is such that $X$. $(u . e)=$ $\lambda^{\prime}(\operatorname{pr}(X)),(\forall X \in \mathfrak{r})$, then it follows that $u \in U\left(\mathfrak{u}^{-}\right)^{\mathfrak{g}_{S}}$, and $\operatorname{ad}(X) u=\left(\lambda^{\prime}-\right.$ $\lambda)(\operatorname{pr}(X)) \cdot u,(\forall X \in \mathfrak{r})$. By Proposition 4.2 of [2], the space $U\left(\mathfrak{u}^{-}\right)^{\mathfrak{g}_{S}}$ is either $\mathbb{C}$, or else it is $\mathbb{C}\left[u_{r}\right]$, for a certain element $u_{r}$. which satisfies $\operatorname{ad}(X) u_{r}=$ $-2 \omega_{\alpha}(X) u_{r}$ (cf. the computation of " $\mu_{r}$ " at the end of the proof of Theorem 4.4).

The heart of the proof of [2] Theorem 4.4 is a proof that $u_{r}^{k} \otimes e_{\lambda}$ is annihilated by $\mathfrak{u}^{+}$if and only if $\lambda=\left(k-\rho^{S}\left(h_{\alpha}\right)\right) \omega_{\alpha}$. It goes through word for word if we replace $u_{r}^{k} \otimes e_{\lambda}$ by $u_{r}^{k} e$ for any $e$ which satisfies (Hol wt $\lambda$ ), provided $u_{r}^{k} e \neq 0$. This proves that $(1) \Longrightarrow(3)$, and that $(3) \Longrightarrow(2)$.

Proof of Proposition 2.1. Proposition 2.1 is a fairly direct application of Theorem 3.1. If $c$ is the Cayley transformation as in [6], one takes $\mathfrak{g}=\mathfrak{s p}(2 n, \mathbb{C})$, and

$$
\begin{gathered}
\mathfrak{p}=\left\{c^{-1}\left(\begin{array}{cc}
X_{0} & 0 \\
X & -{ }^{\top} X_{0}
\end{array}\right) c: X_{0} \in \mathfrak{g l}(n, \mathbb{C}), X \in \operatorname{Mat}_{n}(\mathbb{C}), \text { symmetric }\right\}, \\
\mathfrak{u}^{-}=\left\{c^{-1}\left(\begin{array}{cc}
0 & X \\
0 & 0
\end{array}\right) c: X \in \operatorname{Mat}_{n}(\mathbb{C}), \text { symmetric }\right\} . \\
\mathfrak{r}=\left\{c^{-1}\left(\begin{array}{cc}
X_{0} & 0 \\
0 & -{ }^{\top} X_{0}
\end{array}\right) c: X_{0} \in \mathfrak{g l}(n, \mathbb{C})\right\}, \\
\mathfrak{g}_{S}=\left\{c^{-1}\left(\begin{array}{cc}
X_{0} & 0 \\
0 & -{ }^{\top} X_{0}
\end{array}\right) c: X_{0} \in \mathfrak{s l}(n, \mathbb{C})\right\} .
\end{gathered}
$$

The root $\alpha$ is the unique long simple root of $\mathfrak{s p}(2 n, \mathbb{C})$. The element $c^{-1} h_{\alpha} c$ has a -1 at $n, n$, a 1 at $2 n, 2 n$, and zeros everywhere else, and $\rho^{S}\left(c^{-1} h_{\alpha} c\right)=\frac{n+1}{2}$. Also

$$
\omega_{\alpha} \circ \operatorname{pr}\left[c^{-1}\left(\begin{array}{cc}
X_{0} & 0 \\
X & -{ }^{\top} X_{0}
\end{array}\right) c\right]=-\operatorname{Tr}\left(X_{0}\right) .
$$

What remains is to check that the generator $u_{r}$ for $U\left(\mathfrak{u}^{-}\right)^{\mathfrak{g}_{S}}$ is the element $M_{+}$defined above. This is not difficult. Since $\mathfrak{u}^{-}$is commutative, the algebra $U\left(\mathfrak{u}^{-}\right)$may be identified with the symmetric algebra $S\left(\mathfrak{u}^{-}\right)$. We my identify $X \in \mathfrak{u}^{-}$with the linear form $Y \mapsto \operatorname{Tr}(X Y), \mathfrak{u}^{+} \rightarrow \mathbb{C}$, and thus identify 
$S\left(\mathfrak{u}^{-}\right)$with the algebra of polynomial-functions on $\mathfrak{u}^{+}$. The space of $S L_{n}(\mathbb{C})$ invariant symmetric polynomials on $\mathfrak{u}^{+}$is well-known to be generated by the map $\left(\begin{array}{ll}0 & 0 \\ X & 0\end{array}\right) \mapsto \operatorname{det} X$, and one easily checks that the corresponding element of $S\left(\mathfrak{u}^{-}\right)$is $M_{+}$.

Remark 3.4. The preceding proof consists mainly of explaining what the key players in Boe's general theory are in the special case $\mathfrak{g}=\mathfrak{s} \mathfrak{p}(2 n, \mathbb{C})$. These details are also worked out in detail in Boe's Ph. D. thesis [3].

\section{Longer proof of Proposition 2.1}

The purpose of this section is to give a proof of Proposition 2.1 which is along the lines of the proof given for the case $n=2$ in [6]. Thus, we return to the notation of Section 2: $\mathfrak{g}=\mathfrak{s p}(2 n, \mathbb{C})$, etc.

\subsection{Step 1}

The first step is to show that the weight of a holomorphic and semispherical vector determines the eigenvalue of the Laplace-Beltrami operator acting on that vector, and hence on the entire $U(\mathfrak{g})$-module that it generates. Recall the Laplace-Beltrami operator is a differential operator of degree two which lies in the center of $U(\mathfrak{g})$, and that these conditions determine it uniquely up to scalar. As noted on p. 129 of [6], a theorem of Harish-Chandra implies that this operator is the image under the symmetrization map $\lambda([6]$, p. 128) of an ad-invariant element of $S(\mathfrak{g})$. Such elements of $S(\mathfrak{g})$ may be identified with ad-invariant polynomial-functions on $S(\mathfrak{g})$ using the ad-invariant bilinear form $(X, Y):=\operatorname{Tr}(X Y)$. The Laplace-Beltrami operator may then be normalized to be the operator $\Delta$ corresponding to the polynomial-function $A \mapsto \operatorname{Tr}\left(A^{2}\right)$.

This operator can be written out explicitly in terms of a basis for $\mathfrak{g}$. Write $e_{i, j}$ for the matrix with 1 at $i, j$ and zeros everywhere else. Take $d_{i}=e_{i, i}-e_{n+i, n+i}$ for each $i=1$ to $n$. Then $\left\{d_{i}: 1 \leq i \leq n\right\}$ is a basis for the standard Cartan subalgebra of $\mathfrak{g}$. Write $\left\{d_{i}^{*}: 1 \leq i \leq n\right\}$ for the dual basis. Then the positive roots of $\mathfrak{h}$ in $\mathfrak{g}$ are

$$
\left\{d_{i}^{*}-d_{j}^{*}: 1 \leq i<j \leq n\right\} \cup\left\{d_{i}^{*}+d_{j}^{*}: 1 \leq i<j \leq n\right\} \cup\left\{2 d_{i}^{*}: 1 \leq i \leq n\right\} .
$$

Roots from one of the first two sets above are short and roots from the last are long. For each positive root define $X_{\alpha}$ to be

$$
X_{\alpha}:= \begin{cases}e_{i, j}-e_{n+j, n+i}, & \alpha=d_{i}^{*}-d_{j}^{*}, \\ e_{i, n+j}+e_{j, n+i}, & \alpha=d_{i}^{*}+d_{j}^{*}, \\ e_{i}, n+i, & \alpha=2 d_{i}^{*} .\end{cases}
$$

In all cases define $Y_{\alpha}$ to be the transpose. Then teasing out the definitions, one obtains

$$
\Delta=\lambda\left(\sum_{i=1}^{n} d_{i}^{2}+2 \sum_{\alpha \text { short }} X_{\alpha} Y_{\alpha}+4 \sum_{\alpha \text { long }} X_{\alpha} Y_{\alpha}\right)
$$




$$
\begin{aligned}
& =\sum_{i=1}^{n} d_{i}^{2}+\sum_{\alpha \text { short }}\left(X_{\alpha} Y_{\alpha}+Y_{\alpha} X_{\alpha}\right)+2 \sum_{\alpha \text { long }}\left(X_{\alpha} Y_{\alpha}+Y_{\alpha} X_{\alpha}\right) \\
& =\left(\sum_{i=1}^{n} d_{i}^{2}-\sum_{\alpha} c_{\alpha}\left[X_{\alpha}, Y_{\alpha}\right]\right)+\left(2 \sum_{\alpha} c_{\alpha} X_{\alpha} Y_{\alpha}\right)
\end{aligned}
$$

where the sums are over positive roots $\alpha$ and $c_{\alpha}$ is 2 if $\alpha$ is long and 1 if $\alpha$ is short.

Lemma 4.1. Let $V$ be a $U(\mathfrak{g})$-module generated by a vector $v$ which satisfies (Hol) and (Weight $k$ ). Let $\Delta$ denote the Laplace-Beltrami operator, let

$$
\Delta^{(1)}=\left(\sum_{i=1}^{n} d_{i}^{2}-\sum_{\alpha} c_{\alpha}\left[X_{\alpha}, Y_{\alpha}\right]\right)
$$

and let $\hat{M}_{+}=c M_{+} c^{-1}$, where $M_{+}$is the operator defined on $p .123$ of [6]. Equivalently, $\hat{M}_{+}=\operatorname{det} \hat{R}_{X_{i j}}$, with the same convention as on $p .123$ of [6]. Then

$$
\begin{gathered}
\Delta^{(1)} M_{+}^{r} v=(k+2 r) n(k+2 r-n-1) M_{+}^{r} v \quad(r=0,1,2,3, \ldots), \\
\Delta w=k n(k-n-1) w \quad(\forall w \in V) .
\end{gathered}
$$

Proof. One first checks (4.2) using (Hol), together with Proposition 5 of p. 124 of [6]. Equation (4.3) for $w=v$ then follows since $\Delta-\Delta^{(1)}$ kills any holomorphic vector. Equation (4.3) then holds for all $w$ because $\Delta$ is in the center of $U(\mathfrak{g})$.

Remark 4.4. This also gives a second proof that the $U(\mathfrak{g})$-module generated by a holomorphic vector can contain at most one other linearly independent holomorphic vector, and that such a vector may exist only when the weight of the generator is of the form $-r+\frac{n-1}{2}$, in which case the second linearly independent vector must be of weight $r+2+\frac{n-1}{2}$.

Corollary 4.5. If $v$ and $r$ are as above, and furthermore $k=-r+\frac{n-1}{2}$, then

$$
\left(2 \sum_{\alpha} c_{\alpha} X_{\alpha} Y_{\alpha}\right) \hat{M}_{+}^{r+1} v=0 .
$$

Here the sum may be taken over all positive roots, or only over the roots of $\mathfrak{h}$ in $\mathfrak{R}$, (defined on p. 122 of [6]) since $\hat{M}_{+}^{r+1} v$ satisfies (Weight $r+2+\frac{n-1}{2}$ ).

Proof. If $r, k$, and $n$ stand in this relationship to one another, then $k n(k-n-$ $1)=(k+2 r) n(k+2 r-n-1)$ and our claim follows by subtracting the two equations of the previous corollary. 


\subsection{An isomorphism}

The next step to mimic the argument given at the bottom of p. 131 of [6]. To that end define $u=\hat{M}_{+}^{r} v_{0}$ and $w=\hat{M}_{+}^{r+1} v_{0}$, where $c$ is the Cayley transform. Let $\mathfrak{L}=\operatorname{Span}\left\{\left(\begin{array}{ll}0 & 0 \\ X & 0\end{array}\right) \mid X \in \operatorname{Mat}_{n} \mathbb{C}\right.$, symmetric $\}$. The goal is to prove that $w$ is annihilated by $U(\mathfrak{L}) \subset U(\mathfrak{g})$. If $w$ is zero, then there is nothing to prove. Therefore, one may assume that $w$ is nonzero, in which case clearly $u$ is also nonzero.

Now, recall that $\hat{M}_{+}$is defined as the determinant of a certain matrix with entries in the universal enveloping algebra of $\mathfrak{R}$ (which is a commutative ring). See $[6]$, p. 123. Let us now write this out explicitly. Let $\alpha(i, i)$ be the root such that $2 d_{i}^{*}$ and for $i \neq j$ let $\alpha(i, j)$ be the root $d_{i}^{*}+d_{j}^{*}$ (In particular, $\alpha(i, j)=\alpha(j, i))$. Then

$$
\hat{M}_{+}=\operatorname{det}\left(c_{\alpha(i, j)} X_{\alpha(i, j)}\right),
$$

where $c_{\alpha}$ is defined as above. Now, for each $i, j$ let $\hat{M}_{+}^{(i, j)}$ denote the $i, j$ cofactor in this determinant, (that is, the determinant of the appropriate minor, times the appropriate sign) which is also an element of the universal enveloping algebra of $\mathfrak{R}$.

Identify $\mathfrak{g l}(n, \mathbb{C})$ with $\operatorname{Ad}\left(c^{-1}\right) \mathfrak{k}$ via the map $X_{0} \mapsto\left(\begin{array}{cc}X_{0} & 0 \\ 0 & -{ }^{\top} X_{0}\end{array}\right)$.

Proposition 4.6. The space

$$
\operatorname{Span}\left(\left\{\hat{M}_{+}^{(i, j)} u: 1 \leq i, j \leq n\right\}\right)
$$

is nonzero, and it is an irreducible $\mathfrak{g l}(n, \mathbb{C})$ module. Assume that

$$
\operatorname{Span}\left(\left\{Y_{\alpha} w: \alpha \in \Phi\left(\mathfrak{h}, \mathfrak{u}^{+}\right)\right\}\right)
$$

is nonzero. Then it is an irreducible $\mathfrak{g l}(n, \mathbb{C})$ module which is isomorphic to (4.7).

Proof. Since $w=\hat{M}_{+} u$ can be expressed as an $\mathfrak{R}$-linear combination of elements of (4.7), the fact that (4.7) is nonzero follows from the assumption that $w$ is nonzero. First, as $\mathfrak{s l}(n, \mathbb{C})$-modules, $\mathfrak{R}$ and $\mathfrak{L}$ are both isomorphic to the symmetric square representation, which is irreducible and self-dual. The expansion of the determinant (an $\mathfrak{s l}(n, \mathbb{C})$-invariant) by minors defines an $\mathfrak{s l}(n, \mathbb{C})$ invariant bilinear form between $\mathfrak{R}$ and the space of minors

$$
\operatorname{Span}\left(\left\{\hat{M}_{+}^{(i, j)}: 1 \leq i, j \leq n\right\}\right) \subset U\left(\mathfrak{u}^{+}\right) .
$$

When regarded as $\mathfrak{g l}(n, \mathbb{C})$-modules, $\mathfrak{L}$ remains isomorphic to the dual, $\mathfrak{R}^{*}$, of $\mathfrak{R}$, while the $\mathfrak{s l}(n, \mathbb{C})$-invariant bilinear form between $(4.9)$ and $\mathfrak{R}$ spans a one dimensional $\mathfrak{g l}(n, \mathbb{C})$-module on which $X_{0} \in \mathfrak{g l}(n, \mathbb{C})$ acts by $2 \operatorname{Tr} X_{0}$. It follows that $(4.9)$ is isomorphic, as a $\mathfrak{g l}(n, \mathbb{C})$-module to the twist of $\mathfrak{R}^{*}$, or, equivalently, of $\mathfrak{L}$, by the one dimensional representation $2 \operatorname{Tr}$. 
Now, if $x$ is any semispherical vector $x \in V$ of weight $l$, the mapping $D \rightarrow D x$ is a $\mathfrak{s l}(n, \mathbb{C})$-equivariant linear map $U(\mathfrak{g}) \rightarrow V$. On each irreducible sub- $\mathfrak{g l}(n, \mathbb{C})$ module $W$ of $U(\mathfrak{g})$ it is either trivial or injective, and when injective the image is a sub- $\mathfrak{g l}(n, \mathbb{C})$-module of $V$ isomorphic to the twist of $W$ by $l \mathrm{Tr}$. Thus (4.9) and (4.8) are both isomorphic to the twist of $\mathfrak{L}$ by $(k+2 r+2) \operatorname{Tr}$.

Corollary 4.10. There is a scalar $C$ such that

$$
Y_{\alpha(i, j)} w=C \hat{M}_{+}^{(i, j)} u \quad(\forall 1 \leq i, j \leq n) .
$$

Proof. If (4.8) is trivial, then the statement holds with $C=0$. Otherwise, comparing the $\mathfrak{s l}(n, \mathbb{C})$-invariant bilinear forms on $\mathfrak{R} \times \mathfrak{L}$ and $\mathfrak{R} \times(4.9)$, one sees that the isomorphism $(4.8) \rightarrow(4.7)$ must map $Y_{\alpha(i, j)} w$ to $C \hat{M}_{+}^{(i, j)} u$ for each $i, j$. Now, both (4.8) and (4.7) are contained in $U(\mathfrak{g}) v_{0}=U(\mathfrak{R}) v_{0}$, and it follows from Proposition 4, p. 122 of [6] that this space is multiplicity-free as a $\mathfrak{g l}(n, \mathbb{C})$ module. The result follows.

\subsection{Completion of proof of 2.1}

In view of Proposition 5 of [6] (p. 124), it suffices to prove that the vector $w=\hat{M}_{+}^{r+1}$ satisfies $(\mathrm{Hol})$, i.e., is annihilated by $\mathfrak{L}$. Since $\mathfrak{L}$ is irreducible as a $\mathfrak{s l}(n, \mathbb{C})$-module, and the mapping $X \rightarrow X w$ is $\mathfrak{s l}(n, \mathbb{C})$-equivariant, its kernel is either trivial or all of $\mathfrak{L}$. Hence, it suffices to prove that $w$ is annihilated by a single element of $\mathfrak{L}$. We shall show that $w$ is annihilated by $\sum_{1 \leq i \leq j \leq n} Y_{\alpha(i, j)}$. Suppose not. Then, the constant $C$ in Corollary 4.10 is nonzero. It follows that

$$
\begin{aligned}
0 & =\sum_{\alpha \in \Phi^{+}} c_{\alpha} X_{\alpha} Y_{\alpha} w=\sum_{1 \leq i \leq j \leq n} c_{\alpha(i, j)} X_{\alpha(i, j)} Y_{\alpha(i, j)} w \\
& =C \sum_{1 \leq i \leq j \leq n} c_{\alpha(i, j)} X_{\alpha(i, j)} \hat{M}_{+}^{(i, j)} u=C \hat{M}_{+} u=C w
\end{aligned}
$$

a contradiction.

\section{The other elements of the center}

It is at first surprising that one may prove Proposition 2.1, and hence conjecture 2 of [6] using an argument which references only the Laplace-Beltrami operator, and not any of the other elements of the center of $U(\mathfrak{g})$. However, a hint as to why this is so is already available in Theorem 3 of [6]. We briefly review this result.

The center of the universal enveloping algebra of $\mathfrak{s p}(4, \mathbb{C})$ is a polynomial ring in two generators, one of degree 2 and the other of degree 4 . The degree 2 generator is unique up to scalar, and the degree 4 generator is unique up to scalar modulo the span of the square of the degree 2 generator. Explicit choices for the two generators, $\mathfrak{D}_{2}$ and $\mathfrak{D}_{4}$, are fixed on p. 128 of [6], and it is shown in Theorem 3 that $\mathfrak{D}_{4} \equiv-2 \mathfrak{D}_{2}$, modulo a right ideal $\mathcal{J}$ which annihilates any vector $v$ satisfying (Weight $k$ ) and ( $\mathrm{Hol}$ ). 
The next theorem generalizes this phenomenon. In order to state it, some notation is required. Let $\mathcal{J}$ denote the right ideal in $U(\mathfrak{g})$ generated by $\mathfrak{p}$. Let $\mathcal{Z}$ denote the center of $U(\mathfrak{g})$. Let $\mathfrak{b}$ denote the Borel subalgebra of $\mathfrak{g}$ consisting of all elements $\left(\begin{array}{cc}X_{0} & 0 \\ X & -{ }^{\top} X_{0}\end{array}\right) \in \mathfrak{p}$ such that $X_{0}$ is lower triangular. Let $\rho$ denote half the sum of the roots of $\mathfrak{h}$ in $\mathfrak{b}$. Let $\mathfrak{h}_{0}$ be the kernel of $\operatorname{Tr}$ in $\mathfrak{h}$, and fix $H_{0} \in \mathfrak{h} \backslash \mathfrak{h}_{0}$.

Theorem 5.1. Let $\gamma$ be the isomorphism $\mathcal{Z} \rightarrow S(\mathfrak{h})^{W}$ defined on $p .118$ of [8]. Let $t: S(\mathfrak{h}) \rightarrow S(\mathfrak{h})$ be the $\mathbb{C}$-algebra isomorphism which is given on elements of $\mathfrak{h}$ by $H \mapsto H+\rho(H)$. let $\mathrm{pr}_{1}$ denote projection onto the first factor in the canonical isomorphism $S(\mathfrak{h}) \rightarrow \mathbb{C}\left[H_{0}\right] \oplus \mathfrak{h}_{0} S(\mathfrak{h})$. Then

$$
\mathfrak{D} \equiv \operatorname{pr}_{1} \circ t \circ \gamma(\mathfrak{D}) \quad(\bmod \mathcal{J}) \quad \forall \mathfrak{D} \in \mathcal{Z} .
$$

Proof. This follows from the construction of $\gamma$ given on p. 118 of [8]. Let $\mathcal{I}$ denote the ideal in $U(\mathfrak{g})$ generated by the nilpotent radical of $\mathfrak{b}$. Then $\gamma=$ $t^{-1} \circ \gamma^{\prime}$ where $\gamma^{\prime}(\mathfrak{D})$ is uniquely determined by the fact that $\gamma^{\prime}(\mathfrak{D}) \in U(\mathfrak{h})$ and $\mathfrak{D}-\gamma^{\prime}(\mathfrak{D}) \in \mathcal{I}$ for all $\mathfrak{D} \in U(\mathfrak{g})$. Thus $\mathfrak{D} \equiv \gamma^{\prime}(\mathfrak{D})=t \circ \gamma(\mathfrak{D})(\bmod \mathcal{J})$. Clearly, $\mathcal{J}$ contains both $\mathcal{I}$ and $\mathfrak{h}_{0} S(\mathfrak{h})$.

Thus, the action of $\mathcal{Z}$ on $V$ factors through the projection to a quotient ring which is isomorphic to a polynomial algebra in one generator, and generated by the image of the Laplace-Beltrami operator.

\section{Notation for the Jacobi case}

First, let us set up some notation. The treatment of Jacobi forms in this paper is influenced by [1] as well as [6] and [7]. Fix integers $n$ and $j$ for the remainder of the paper.

For a ring $R$, let $\operatorname{sym}_{j}^{2}(R)$ for the space of symmetric $j \times j$ matrices with entries in $R$.

\subsection{Jacobi group}

Define the Jacobi group $G^{(n, j)}$ to be the following subgroup of $S p(2 n+2 j)$ :

$$
\left\{\left(\begin{array}{cccc}
I_{n} & 0 & 0 & { }^{\top} u \\
v & I_{j} & u & \zeta \\
0 & 0 & I_{n} & -{ }^{\top} v \\
0 & 0 & 0 & I_{j}
\end{array}\right)\left(\begin{array}{cccc}
A & 0 & B & 0 \\
0 & I_{j} & 0 & 0 \\
C & 0 & D & 0 \\
0 & 0 & 0 & I_{j}
\end{array}\right): u, v \in \operatorname{Mat}_{j \times n}, \zeta+u^{\top} v \in \operatorname{sym}_{j}^{2},\left(\begin{array}{cc}
A & B \\
C & D
\end{array}\right) \in S p(2 n)\right\} .
$$

It is convenient to identify

$$
\left(\begin{array}{cc}
A & B \\
C & D
\end{array}\right) \in S p(2 n) \text { with }\left(\begin{array}{cccc}
A & 0 & B & 0 \\
0 & I_{j} & 0 & 0 \\
C & 0 & D & 0 \\
0 & 0 & 0 & I_{j}
\end{array}\right) \in G^{(n, j)} \text {, and }
$$




$$
(v, u, \zeta) \text { with }\left(\begin{array}{cccc}
I_{n} & 0 & 0 & \top^{\top} u \\
v & I_{j} & u & \zeta \\
0 & 0 & I_{n} & -{ }^{\top} v \\
0 & 0 & 0 & I_{j}
\end{array}\right) .
$$

Doing so equips $H^{(n, j)}:\left\{(v, u, \zeta) \in \operatorname{Mat}_{j \times n} \times \operatorname{Mat}_{j \times n} \times \operatorname{Mat}_{j \times j}: \zeta+u^{\top} v \in\right.$ $\left.\operatorname{sym}_{j}^{2}\right\}$ with the structure of a Heisenberg group, with operation

$$
\left(v_{1}, u_{1}, w_{1}\right) \cdot\left(v_{2}, u_{2}, w_{2}\right)=\left(v_{1}+v_{2}, u_{1}+u_{2}, w_{1}+w_{2}+v_{1}^{\top} u_{2}+u_{1}^{\top} v_{2}\right) .
$$

\subsection{Symmetric space $\mathcal{H}^{(n, j)}$}

Let $\mathcal{H}_{n}$ denote the Siegel upper half-plane of genus $n$, i.e., the space of all $\tau=X+i Y \in \operatorname{sym}_{n}^{2}(\mathbb{C})$ with $X, Y \in \operatorname{sym}_{n}^{2}(\mathbb{R})$ and $Y$ positive definite. The group $G^{(n, j)}(\mathbb{R})$ acts on $\mathcal{H}^{(n, j)}:=\mathcal{H}^{n} \times \operatorname{Mat}_{j \times n}(\mathbb{C})$ by the formulae

$$
\begin{aligned}
\left(\begin{array}{ll}
A & B \\
C & D
\end{array}\right) \cdot(\tau, z) & =\left((A \tau+B)(C \tau+D)^{-1}, z(C \tau+D)^{-1}\right), \\
(v, u, \zeta) \cdot(\tau, z) & =(\tau, z+u+\tau v) .
\end{aligned}
$$

The stabilizer of $\left(i I_{n}, 0\right)$ is $U(n) \times Z(\mathbb{R})$, where $Z=\left\{(0,0, \zeta): \zeta \in \operatorname{sym}_{j}^{2}\right\}$ is the center of $G^{(n, j)}$.

Given $\mathcal{M} \in \operatorname{sym}_{j}^{2}(\mathbb{C})$ define $e^{\mathcal{M}}: \operatorname{Mat}_{j \times j}(\mathbb{C}) \rightarrow \mathbb{C}$ by $e^{\mathcal{M}}(A)=e^{2 \pi i \operatorname{Tr}(\mathcal{M} A)}$. Observe that $e^{\mathcal{M}}(A)=e^{\mathcal{M}}\left({ }^{\top} A\right)$ for all $A \in \operatorname{Mat}_{j \times j}(\mathbb{C})$.

\subsection{Covering groups}

Let $\widetilde{S p}(2 n, \mathbb{R})$ be the metaplectic double cover of $S p(2 n, \mathbb{R})$. Choose a branch of the square root, and define

$$
J_{\frac{1}{2}}\left(\left(\begin{array}{cc}
A & B \\
C & D
\end{array}\right), \tau\right):=\operatorname{det}(C \tau+D)^{-\frac{1}{2}}, \quad \sigma\left(g_{1}, g_{2}\right)=\frac{J_{\frac{1}{2}}\left(g_{1} g_{2}, \tau\right)}{J_{\frac{1}{2}}\left(g_{1}, g_{2} \tau\right) J_{\frac{1}{2}}\left(g_{2}, \tau\right)}
$$

Then one may identify $\widetilde{S p}(2 n, \mathbb{R})$ with the set $S p(2 n, \mathbb{R}) \times\{ \pm 1\}$, equipped with multiplication $\left(g_{1}, \varepsilon_{1}\right)\left(g_{2}, \varepsilon_{2}\right)=\left(g_{1} g_{2}, \sigma\left(g_{1}, g_{2}\right) \varepsilon_{1} \varepsilon_{2}\right)$, as in [6]. However, the topology on $\widetilde{S p}(2 n, \mathbb{R})$ is such that multiplication is continuous, and hence disagrees with that of $S p(2 n, \mathbb{R}) \times\{ \pm 1\}$.

Define an action of $\widetilde{S p}(2 n, \mathbb{R})$ on $H_{\mathbb{R}}^{(n, j)}$ by composing the usual action of $S p(2 n, \mathbb{R})$ with the projection $\widetilde{S p}(2 n, \mathbb{R}) \rightarrow S p(2 n, \mathbb{R})$, and let $\widetilde{G}_{\mathbb{R}}^{(n, j)}$ be the semidirect product $\widetilde{S p}(2 n, \mathbb{R}) \rtimes H_{\mathbb{R}}^{(n, j)}$, defined using this action. The function

$$
J_{\frac{1}{2}}\left(\left(\left(\begin{array}{cc}
A & B \\
C & D
\end{array}\right), \varepsilon\right), \tau\right)=J_{\frac{1}{2}}\left(\left(\begin{array}{cc}
A & B \\
C & D
\end{array}\right), \tau\right) \varepsilon
$$

satisfies the cocycle condition

$$
J_{\frac{1}{2}}\left(g_{1} g_{2}, \tau\right)=J_{\frac{1}{2}}\left(g_{1}, g_{2} \tau\right) J_{\frac{1}{2}}\left(g_{2}, \tau\right) \quad\left(\forall g_{1}, g_{2} \in \widetilde{S p}(2 n, \mathbb{R}), \tau \in \mathcal{H}_{n}\right) .
$$




\subsection{Cocycle and slash operator}

Fix $k \in \mathbb{Z}$ and $\mathcal{M} \in \operatorname{sym}_{j}^{2} \frac{1}{2} \mathbb{Z}$. For $\left(\left(\begin{array}{cc}A & B \\ \hline & D\end{array}\right), \varepsilon\right) \in \widetilde{S p}(2 n, \mathbb{R}),(v, u, \zeta) \in H_{\mathbb{R}}^{(n, j)}$, and $(\tau, z) \in \mathcal{H}^{(n, j)}$, define

$$
\begin{aligned}
& j_{\frac{k}{2}, \mathcal{M}}(g(v, u, \zeta),(\tau, z)) \\
= & J_{\frac{1}{2}}(g, \tau)^{k} e^{\mathcal{M}}\left(\zeta+(v \tau+2 z+u)^{\top} v-(z+u+v \tau)(C \tau+D)^{-1} C(z+u+v \tau)\right) .
\end{aligned}
$$

Then $j_{\frac{k}{2}, \mathcal{M}}$ satisfies the cocycle condition

$$
\begin{aligned}
j_{\frac{k}{2}, \mathcal{M}}\left(g_{1} g_{2}, \underline{\mathrm{x}}\right) & =j_{\frac{k}{2}, \mathcal{M}}\left(g_{1}, g_{2} \underline{\mathrm{x}}\right) j_{\frac{k}{2}, \mathcal{M}}\left(g_{2}, \underline{\mathrm{x}}\right) \\
& \left(\forall g_{1}, g_{2} \in \widetilde{G}_{\mathbb{R}}^{(n, j)}, \underline{\mathrm{x}} \in \mathcal{H}^{n} \times \operatorname{Mat}_{j \times n}(\mathbb{C})\right) .
\end{aligned}
$$

Now, for $f: \mathcal{H}^{(n, j)} \rightarrow \mathbb{C}$ and $g \in \widetilde{G}_{\mathbb{R}}^{(n, j)}$ define $\left.f\right|_{\frac{k}{2}, \mathcal{M}} g: \mathcal{H}^{(n, j)} \rightarrow \mathbb{C}$ by

$$
\left(\left.f\right|_{\frac{k}{2}, \mathcal{M}} g\right)(\underline{\mathrm{x}}):=j_{\frac{k}{2}, \mathcal{M}}(g, \underline{\mathrm{x}}) f(g \underline{\mathrm{x}}) \quad\left(\underline{\mathrm{x}} \in \mathcal{H}^{(n, j)}\right) .
$$

\subsection{Meromorphic Jacobi forms}

Let $j_{\frac{k}{2}, \mathcal{M}}^{0}: \widetilde{G}_{\mathbb{R}}^{(n, j)} \times \mathcal{H}^{(n, j)} \rightarrow \mathbb{C}$ be defined by the same formula as $j_{\frac{k}{2}, \mathcal{M}}$ with the factor $\varepsilon^{k}$ omitted. To define Jacobi forms, one chooses a discrete subgroup $\Gamma \subset G_{\mathbb{R}}^{(n, j)}$, and a function $\chi: \Gamma \rightarrow \mathbb{C}$ such that $(\gamma, \underline{\mathrm{x}}) \mapsto \chi(\gamma) j_{\frac{k}{2}, \mathcal{M}}^{0}(\gamma, \underline{\mathrm{x}})$ is a cocycle. In this case $\tilde{\chi}((\gamma, \varepsilon)):=\chi(\gamma) \varepsilon$ is a character of the preimage $\widetilde{\Gamma}$ of $\Gamma$ in $\widetilde{G}_{\mathbb{R}}^{(n, j)}$. Then a meromorphic Jacobi form of weight $\frac{k}{2}$, index $\mathcal{M}$, and multiplier $\chi$ is a meromorphic function $f: \mathcal{H}^{(n, j)} \rightarrow \mathbb{C}$ which satisfies

$$
j_{\frac{k}{2}, \mathcal{M}}^{0}(\gamma, \underline{\mathrm{x}}) \chi(\gamma) f(\gamma \underline{\mathrm{x}})=f(\underline{\mathrm{x}}) \quad\left(\forall \gamma \in \Gamma, \underline{\mathrm{x}} \in \mathcal{H}^{(n, j)}\right)
$$

One might equivalently describe it as being invariant under the right-action of $\widetilde{\Gamma}$ on functions $\mathcal{H}^{(n, j)} \rightarrow \mathbb{C}$ defined by

$$
\left(\left.f\right|_{\frac{k}{2}, \mathcal{M}, \tilde{\chi}} \gamma\right)(\underline{\mathrm{x}}):=j_{\frac{k}{2}, \mathcal{M}}(\gamma, \underline{\mathrm{x}}) \widetilde{\chi}(\gamma) f(\gamma \underline{\mathrm{x}}) \quad\left(\gamma \in \widetilde{\Gamma}, \underline{\mathrm{x}} \in \mathcal{H}^{(n, j)}\right) .
$$

7. Main result, a la Choie-Kim

Definition 7.1. Take $\mathcal{M} \in \operatorname{sym}_{j}^{2}(\mathbb{R})$, positive definite. Define $\frac{\partial}{\partial \tau}$ to be the $n \times n$ matrix with $r, s$ entry $\left(1+\delta_{r, s}\right) \frac{\partial}{\partial \tau_{r s}}$, and $\frac{\partial}{\partial z}$ the $j \times n$ matrix with $r, s$ entry $\frac{\partial}{\partial z_{r s}}$. Finally, write $|\mathcal{M}|$ for the determinant of $\mathcal{M}$ and $\widetilde{\mathcal{M}}$ for the classical adjoint of $\mathcal{M}$, i.e., the matrix of cofactors satisfying $\mathcal{M}^{-1}=|\mathcal{M}|^{-1} \widetilde{\mathcal{M}}$. Then

$$
L_{\mathcal{M}}:=\operatorname{det}\left(4 \pi i|\mathcal{M}| \frac{\partial}{\partial \tau}-\frac{\partial}{\partial z} \widetilde{\mathcal{M}} \frac{\partial}{\partial z}\right) .
$$

Theorem 7.2. Let $f$ be a meromorphic Jacobi form of weight $-r+\frac{n+j+1}{2}$ and index $\mathcal{M}$. Then $L_{\mathcal{M}}^{k} \cdot f$ is a meromorphic Jacobi form of weight $r+\frac{n+j+1}{2}$ and index $\mathcal{M}$. 
Proof. Theorem 7.2 can actually be deduced from Proposition 2.1 and the results of [6]. First, define the extension by $\boldsymbol{e}^{\mathcal{M}}$ of $f$

$$
\operatorname{ext}_{\mathcal{M}} f\left(\begin{array}{cc}
\tau & \top \\
z & \tau^{\prime}
\end{array}\right)=f(\tau, z) e^{\mathcal{M}}\left(\tau^{\prime}\right) \quad\left(\tau \in \mathcal{H}_{n}, z \in \operatorname{Mat}_{j \times n} \mathbb{C}, \tau^{\prime} \in \mathcal{H}_{j}\right)
$$

Clearly, meromorphicity of $f$ implies that of $\operatorname{ext}_{\mathcal{M}} f$. Further, it follows from a determinant identity given on p. 86 of [7] that

$$
\mathbb{D}^{l} \operatorname{ext}_{\mathcal{M}} f=\left[(4 \pi i)^{n-j} \operatorname{det} \mathcal{M}^{n-1}\right]^{l} \operatorname{ext}_{\mathcal{M}} L_{\mathcal{M}}^{l} f
$$

for all $l \in \mathbb{N}$. Next, define

$$
\widetilde{\sigma}_{k}: C^{\infty}\left(\mathcal{H}_{n+j}\right) \rightarrow C^{\infty}(\widetilde{S} p(2 n+2 j, \mathbb{R})) .
$$

By

$$
\tilde{\sigma}_{k} f(g)=\left[\left.f\right|_{k} g\right]\left(i I_{n+j}\right)=J_{\frac{1}{2}}\left(g, i I_{n+j}\right) f\left(g \cdot i I_{n+j}\right) .
$$

Then for any $f_{1} \in C^{\infty}\left(\mathcal{H}_{n+j}\right)$,

$$
\widetilde{\sigma}_{k} f_{1}=\sigma_{k} F_{1}, \quad \text { where } F_{1}(\tau)=\operatorname{det} Y^{k / 2} f(\tau),
$$

and $\sigma_{k}$ is defined as in [6]. The image of $\sigma_{k}$ is the space

$$
C^{\infty}(\widetilde{S p}(2 n+2 j, \mathbb{R}))_{\left(\widetilde{U}(n+j), \operatorname{det}^{k}\right)}
$$

consisting of all $\phi \in C^{\infty}(\widetilde{S p}(2 n+2 j, \mathbb{R}))$ which satisfy

$$
\phi(g \kappa)=\phi(g) J_{\frac{1}{2}}\left(\kappa, i I_{n+j}\right)^{2 k} \quad(\forall g \in \widetilde{S p}(2 n+2 j, \mathbb{R}), \kappa \in \widetilde{U}(n+j)) .
$$

If $k \in \mathbb{Z}$ this takes the more convenient form

$\phi\left(g\left(\begin{array}{cc}A & -B \\ B & A\end{array}\right)\right)=\phi(g) \operatorname{det}(A+B i)^{k}(\forall g \in S p(2 n+2 j, \mathbb{R}), A+B i \in U(n+j))$.

As shown in $[6]$ the action of $M_{+} \in U(\mathfrak{s p}(2 n+2 j, \mathbb{C}))$ on $C^{\infty}(\widetilde{S p}(2 n+2 j, \mathbb{R}))$ $\operatorname{maps} C^{\infty}(\widetilde{S p}(2 n+2 j, \mathbb{R}))_{\left(\widetilde{U}(n+j), \operatorname{det}^{k}\right)}$ to $C^{\infty}(\widetilde{S p}(2 n+2 j, \mathbb{R}))_{\left(\widetilde{U}(n+j), \operatorname{det}^{k+2}\right)}$ for each $k \in \frac{1}{2} \mathbb{Z}$. This induces operators

$$
\mathbb{M}_{k}:=\widetilde{\sigma}_{k+2}^{-1} \circ M_{+} \circ \widetilde{\sigma}_{k}: C^{\infty}\left(\mathcal{H}_{n+j}\right) \rightarrow C^{\infty}\left(\mathcal{H}_{n+j}\right) \quad\left(k \in \frac{1}{2} \mathbb{Z}\right) .
$$

One may also define $\mathbb{M}_{k}^{l}:=\widetilde{\sigma}_{k+2 l}^{-1} \circ M_{+}^{l} \circ \widetilde{\sigma}_{k}=\mathbb{M}_{k+2 l-2} \circ \cdots \circ \mathbb{M}_{k+2} \circ \mathbb{M}_{k}$ for each $k \in \frac{1}{2} \mathbb{Z}, l \in \mathbb{N}$.

Lemma 7.3. Suppose that $f \in C^{\infty}\left(\mathcal{H}_{n+j}\right)$ and $\mathbb{M}_{k}^{l} f$ are both meromorphic. Then

$$
\mathbb{M}_{k}^{l} f=2^{l n} \mathbb{D}^{l} f .
$$

Proof. We expand slightly on the proof given on p. 126 of [6]. Write $\tau \in \mathcal{H}_{n}$ as $X+i Y$ and say that a function is "simple nonmeromorphic" if it is equal to a meromorphic function times a rational function of $Y$ with the property that the degree of the denominator exceeds that of the numerator. Note that the zero function is both meromorphic and simple nonmeromorphic, and is the only function with both properties. 
It follows from results of Harris [9] that the operator $\mathbb{M}_{k}$ can be realized explicitly as multiplication by $(\operatorname{det} Y)^{-\frac{n+j-1}{2}+k}$ followed by $2^{n} \mathbb{D}$, and then multiplication by $(\operatorname{det} Y)^{\frac{n+j-1}{2}-k}$. This implies that $\mathbb{M}_{k} f-2^{n} \mathbb{D} f$ is a simple nonmeromorphic function for any meromorphic function $f$, and that $\mathbb{M}_{k}$ applied to any simple nonmeromorphic function is again simple nonmeromorphic. Thus, for $f$ meromorphic $\mathbb{M}_{k}^{l} f-2^{n l} \mathbb{D}^{l} f$ is simple nonmeromorphic. If $\mathbb{M}_{k}^{l} f$ is meromorphic, then $\mathbb{M}_{k}^{l} f-2^{n l} \mathbb{D}^{l} f$ is also meromorphic, and therefore zero. let

Let $\operatorname{Mer}\left(\mathcal{H}_{n+j}\right)$ denote the space of meromorphic functions $\mathcal{H}_{n+j} \rightarrow \mathbb{C}$ and

$$
\begin{aligned}
\operatorname{Mer}(\widetilde{S p}(2 n+2 j, \mathbb{R}))_{\left(\widetilde{U}(n+j), \operatorname{det}^{k}\right)}: & \widetilde{\sigma}_{k}\left[\operatorname{Mer}\left(\mathcal{H}_{n+j}\right)\right] \\
& \subset C^{\infty}(\widetilde{S p}(2 n+2 j, \mathbb{R}))_{\left(\widetilde{U}(n+j), \operatorname{det}^{k}\right)} .
\end{aligned}
$$

Now, as explained in [6], pp. 124-127, it follows from Proposition 2.1 that $\mathbb{M}_{-r+\frac{n+j+1}{2}}^{r}$ maps $\operatorname{Mer}\left(\mathcal{H}_{n+j}\right)$ to $\operatorname{Mer}\left(\mathcal{H}_{n+j}\right)$, and therefore coincides with a scalar multiple of $\mathbb{D}^{r}$ on $\operatorname{Mer}\left(\mathcal{H}_{n+j}\right)$.

Now, let $\Lambda$ denote the right-action of $\widetilde{S p}(n, \mathbb{R})$ on $C^{\infty}(\widetilde{S p}(n, \mathbb{R})$ given by $[\Lambda(g) f](h)=f(g h)$. A straightforward calculation shows that

$$
\left(\left.\operatorname{ext}_{\mathcal{M}} f_{1}\right|_{k} g\right)=\operatorname{ext}_{\mathcal{M}}\left(\left.f_{1}\right|_{k, \mathcal{M}} g\right) \quad\left(\forall g \in \widetilde{G}_{\mathbb{R}}^{(n, j)}, f_{1} \in C^{\infty}\left(\mathcal{H}^{(n, j)}\right)\right) .
$$

So

$$
\begin{aligned}
\left.f\right|_{k, \mathcal{M}} \gamma=\tilde{\chi}(\gamma) f & \left.\Longleftrightarrow\left(\operatorname{ext}_{\mathcal{M}} f\right)\right|_{k} \gamma=\tilde{\chi}(\gamma) f \\
& \Longleftrightarrow \Lambda(\gamma) \widetilde{\sigma}_{k}\left(\operatorname{ext}_{\mathcal{M}} f\right)=\tilde{\chi}(\gamma) \widetilde{\sigma}_{k}\left(\operatorname{ext}_{\mathcal{M}} f\right)
\end{aligned}
$$

Now it's clear that the action of $\widetilde{S p}(2 n+2 j, \mathbb{R})$ on $C^{\infty}(\widetilde{S p}(2 n+2 j, \mathbb{R}))$ via $\Lambda$ commutes with the action of $U(\mathfrak{s p}(2 n+2 j, \mathbb{C}))$ on the right. Hence

$$
\Lambda(\gamma) M_{+}^{r} \widetilde{\sigma}_{k}\left(\operatorname{ext}_{\mathcal{M}} f\right)=\tilde{\chi}(\gamma) M_{+}^{r} \widetilde{\sigma}_{k}\left(\operatorname{ext}_{\mathcal{M}} f\right),
$$

and so

$$
\left[\left.\left(\mathbb{M}_{k}^{r} \operatorname{ext}_{\mathcal{M}} f\right)\right|_{k+2 r} \gamma\right]=\tilde{\chi}(\gamma)\left(\mathbb{M}_{k}^{r} \operatorname{ext}_{\mathcal{M}} f\right) .
$$

If $k=-r+\frac{n+j+1}{2}$, then Proposition 2.1 implies that $\left(\mathbb{M}_{k}^{r} \operatorname{ext}_{\mathcal{M}} f\right)$ is meromorphic, and therefore one may replace $\mathbb{M}_{k}^{r}$ be $2^{n r} \mathbb{D}^{r}$. Then the result follows from the relationship between $\mathbb{D}$, $\operatorname{ext}_{\mathcal{M}}$ and $L_{\mathcal{M}}$ already mentioned.

\section{Alternate approach, a la Bump-Choie}

In the last section we showed that Theorem 7.2, which is essentially a version of Bol's result for Jacobi forms, may be proved by extending Jacobi forms to functions on $\mathcal{H}_{n+j}$ which satisfy a certain equivariance property and applying the same results used to prove Bol's result for Siegel modular forms, Conjecture 1 of $[6]$. 
One may ask whether it is also possible to prove Theorem 7.2 by developing a version of Proposition 2.1 for Jacobi forms, which reduces Theorem 7.2 to a statement regarding actions of the Lie algebra of the Jacobi group, and then proving that statement. In this section, we perform a translation for Jacobi forms, similar to the one given in [6] for Siegel modular forms, and then state and prove a result which can be regarded as a version of 2.1 for Jacobi forms. This approach will fall short of a second proof of Theorem 7.2 , because no analogue of Lemma 7.3 will be proved. In other words, we shall show that a certain differential operator which arises naturally from consideration of the universal enveloping algebra exhibits the "recovery" property characteristic of Bol's result, but we shall not prove a result relating this operator back to the more classical heat operator $L_{\mathcal{M}}$ considered in [7].

\subsection{Liftings to the Jacobi group}

For $k \in \frac{1}{2} \mathbb{Z}, \mathcal{M} \in \operatorname{sym}_{j}^{2} \frac{1}{2} \mathbb{Z}$, and $f: \mathcal{H}^{(n, j)} \rightarrow \mathbb{C}$, define

$$
\left[\varphi_{k, \mathcal{M}} f\right](g):=\left(\left.f\right|_{k, \mathcal{M}} g\right)\left(i I_{n}, 0\right)
$$

Clearly, for any function $f$, the function $\left[\varphi_{k, \mathcal{M}} f\right]$ satisfies

$$
\begin{aligned}
& {\left[\varphi_{k, \mathcal{M}} f\right]\left(g(0,0, \zeta)\left(\begin{array}{cc}
A & B \\
-B & A
\end{array}\right)\right)=e^{\mathcal{M}}(\zeta) \varepsilon^{2 k}, \quad \operatorname{det}(A+i B)^{k}\left[\varphi_{k, \mathcal{M}} f\right](g)} \\
& \left(\forall g \in \widetilde{G}_{\mathbb{R}}^{(n, j)}, \zeta \in \operatorname{sym}_{j}^{2}(\mathbb{R}),(A+i B, \varepsilon) \in \widetilde{U}(n)\right) .
\end{aligned}
$$

Moreover, if $f$ satisfies $(6.1)$, then $\varphi_{k, \mathcal{M}} f$ satisfies

$$
\left[\varphi_{k, \mathcal{M}} f\right](\gamma g)=\widetilde{\chi}^{-1}(\gamma)\left[\varphi_{k, \mathcal{M}} f\right](g) \quad\left(\forall \gamma \in \widetilde{\Gamma}, g \in \widetilde{G}_{\mathbb{R}}^{(n, j)}\right) .
$$

\subsection{Relating holomorphicity to the action of the Lie algebra}

Let $\mathfrak{g}^{(n, j)}$ denote the Lie algebra of $G^{(n, j)}$. It consists of all matrices of the form

$$
\left(\begin{array}{cccc}
A & 0 & B & { }^{\top} u \\
v & 0 & u & Z \\
C & 0 & -{ }^{\top} A & -{ }^{\top} v \\
0 & 0 & 0 & 0
\end{array}\right), A \in \operatorname{Mat}_{n \times n}, B, C \in \operatorname{sym}_{n}^{2}, u, v \in \operatorname{Mat}_{j \times n}, Z \in \operatorname{sym}_{j}^{2} .
$$

Identify

$$
\left(\begin{array}{cccc}
A & 0 & B & 0 \\
0 & 0 & 0 & 0 \\
C & 0 & -{ }^{\top} A & \\
0 & 0 & 0 & 0
\end{array}\right) \text { with }\left(\begin{array}{cc}
A & B \\
C & -{ }^{\top} A
\end{array}\right) \in \mathfrak{s p}(2 n), \text { and }
$$




$$
\left(\begin{array}{cccc}
0 & 0 & 0 & \top u \\
v & 0 & u & Z \\
0 & 0 & 0 & -{ }^{\top} v \\
0 & 0 & 0 & 0
\end{array}\right) \text { with }[u, v, Z] \in \operatorname{Mat}_{j \times n} \times \operatorname{Mat}_{j \times n} \times \operatorname{sym}_{j}^{2}
$$

thus inducing a bracket on $\mathfrak{h}^{(n, j)}:=\operatorname{Mat}_{j \times n} \times \operatorname{Mat}_{j \times n} \times \operatorname{sym}_{j}^{2}$,

$$
\left[\left[u_{1}, v_{1}, Z_{1}\right],\left[u_{1}, v_{2}, Z_{1}\right]\right]=\left[0,0,-u_{1}^{\top} v_{2}-v_{2}^{\top} u_{1}+u_{2}^{\top} v_{1}+v_{1}^{\top} u_{2}\right]
$$

(A triple in $\mathfrak{h}^{(n, j)}$ will be written with brackets to avoid being mistaken for an element of $\left.\mathcal{H}^{(n, j)}\right)$.

Next, let

$$
c_{n}=\left(\begin{array}{ll}
\frac{1}{1+i} I_{n} & \frac{-i}{1+i} I_{n} \\
\frac{1}{1+i} I_{n} & \frac{i}{1+i} I_{n}
\end{array}\right)
$$

be the Cayley transform for $S p(2 n)$ (which we identify with an element of $\left.G_{\mathbb{C}}^{(n, j)}\right)$ and define an action of $U\left(\mathfrak{g}_{\mathbb{C}}^{(n, j)}\right)$ on smooth functions $\widetilde{G}_{\mathbb{R}}^{(n, j)} \rightarrow \mathbb{C}$ by twisted right translation

$$
R^{c_{n}}(X) \phi(\widetilde{g}):=\lim _{t \rightarrow 0} \frac{1}{t}\left(\phi\left(\widetilde{g} \exp \left(t c_{n}^{-1} X c_{n}\right)\right)-\phi(\widetilde{g})\right), \quad c_{n}^{-1} X c_{n} \in \mathfrak{g}_{\mathbb{R}}^{(n, j)},
$$

extended to all of $\mathfrak{g}_{\mathbb{C}}^{(n, j)}$ by $\mathbb{C}$-linearity.

Proposition 8.3. A smooth function $f: \mathcal{H}^{(n, j)} \rightarrow \mathbb{C}$ is holomorphic if and only if the corresponding function $\varphi_{k, \mathcal{M}} f$ is annihiliated by $R^{c_{n}}(X)$ for all $X$ of the form

$$
\left(\begin{array}{cc}
0 & 0 \\
X_{0} & 0
\end{array}\right), X_{0} \in \operatorname{sym}_{n}^{2}(\mathbb{R}), \quad[\lambda, 0,0], \lambda \in \operatorname{Mat}_{j \times n}(\mathbb{R}) .
$$

Proof. Write $\underline{x} \in \mathcal{H}^{(n, j)}$ as $(\tau, Z)$ with $\tau \in \mathcal{H}^{n}$ and $Z \in \operatorname{Mat}_{j \times n}(\mathbb{C})$. The first goal is to show that $f: \mathcal{H}^{(n, j)} \rightarrow \mathbb{C}$ is holomorphic in the variable $Z$ if and only if $\varphi_{k, \mathcal{M}} f$ is annihilated by $R^{c_{n}}([\lambda, 0,0])$ for every $\lambda \in \operatorname{Mat}_{j \times n}(\mathbb{R})$. Write $Z=\left(z_{r s}\right)_{r=1 s=1}^{j} \in \operatorname{Mat}_{j \times n}(\mathbb{C})$ as $U+i V$, where $U=\left(u_{r s}\right)_{r=1 s=1}^{j} \underset{n}{n}$ and $V=\left(v_{r s}\right)_{r=1 s=1}^{j}$ are in $\operatorname{Mat}_{j \times n}(\mathbb{R})$. A function is holomorphic in $Z$ if and only if it satisfies:

$$
\frac{\partial f}{\partial z_{r s}}=\frac{\partial f}{\partial u_{r s}}+i \frac{\partial f}{\partial v_{r s}}=0 \quad(\forall 1 \leq r \leq j, 1 \leq s \leq n) .
$$

Form the matrix $\frac{\partial f}{\partial Z}=\left(\frac{\partial f}{\partial z_{r s}}\right)_{r=1 s=1}^{j}$. Then (8.4) is equivalent to

$$
\frac{\partial f}{\partial Z}=0, \quad \text { or } \frac{\partial f}{\partial Z} \cdot A=0 \text { for any given } A \in G L(n, \mathbb{C}) .
$$

Now let $\lambda_{r s}^{*} \in \mathfrak{h}_{\mathbb{R}}^{(n, j)}$ be the element $\left[e_{r s}, 0,0\right]$ where $e_{r s}$ is the matrix with a 1 at the $r s$ position and zeros everywhere else. Also define $\mu_{r s}^{*}$ to be $\left[0, e_{r s}, 0\right]$. 
Define the usual (untwisted) action of $\mathfrak{g}_{\mathbb{R}}^{(n, j)}$ on functions $\widetilde{G}_{\mathbb{R}}^{(n, j)} \rightarrow \mathbb{C}$ by

One checks that

$$
R(X) f(\widetilde{g})=\left.\frac{d}{d t} f(\widetilde{g} \exp (t X))\right|_{t=0} .
$$

$$
\begin{aligned}
& (\lambda, \mu, \zeta) g_{0} \exp \left(t \lambda_{r s}^{*}\right) \cdot(i, 0)=\left(g_{0} \cdot i, \mu+\lambda g_{0} \cdot i+i t \lambda_{r s}^{*}(C i+D)^{-1}\right), \\
& (\lambda, \mu, \zeta) g_{0} \exp \left(t \mu_{r s}^{*}\right) \cdot(i, 0)=\left(g_{0} \cdot i, \mu+\lambda g_{0} \cdot i+t \mu_{r s}^{*}(C i+D)^{-1}\right)
\end{aligned}
$$

for all $(\lambda, \mu, \zeta) \in H_{\mathbb{R}}^{(n, j)}, g_{0}=\left(\begin{array}{c}* \\ C\end{array} \stackrel{*}{D}\right) \in \widetilde{S p}(2 n, \mathbb{R}), t \in \mathbb{R}, 1 \leq r \leq j, 1 \leq s \leq n$. It follows that $(8.5)$, with $A=(C i+D)^{-1}$, is equivalent to

$$
R\left(\mu_{r s}^{*}\right) f+i R\left(\lambda_{r s}^{*}\right) f=0 \quad(\forall 1 \leq r \leq j, 1 \leq s \leq n) .
$$

One checks that $c_{n}\left(\mu_{r s}^{*}+i \lambda_{r s}^{*}\right) c_{n}^{-1}=-2 \lambda_{r s}^{*}$, and this proves that $f$ is holomorphic in $Z$ if and only if it is annihilated by $R^{c_{n}}\left(\lambda_{r s}^{*}\right)$ for all $1 \leq r \leq j, 1 \leq s \leq n$.

Suppose this to be the case. Then $f$ is holomorphic on $\overline{\mathcal{H}}^{n} \times \operatorname{Mat}_{j \times n}(\overline{\mathbb{C}})$ if and only if $\tau \mapsto f(\tau, \mu+\lambda \tau)$ is holomorphic for each fixed $\lambda, \mu \in \operatorname{Mat}_{j \times n}(\mathbb{R})$. Thus, the proposition is reduced to its analogue from the Siegel case, proved in [9], 2.3 .1 (cf. [6], p. 126).

\subsection{A Jacobi analogue of Proposition 2.1}

Theorem 8.7. Let $V$ be $a \mathfrak{g}_{\mathbb{C}}^{(n, j)}$-module. Say that $v \in V$ is holomorphic if

$(\mathrm{Hol}-\mathrm{Jac})$

$$
[\lambda, 0,0] v=\left(\begin{array}{ll}
I & 0 \\
X & I
\end{array}\right) v=0
$$

that $v$ is of index $\mathcal{M}$ if

(ind $\mathcal{M}) \quad[0,0, \zeta] \cdot v=2 \pi i \operatorname{Tr}(\zeta \mathcal{M}) \cdot v \quad \forall \zeta \in \operatorname{Mat}_{j \times j} \mathbb{C}$

and that $v$ is of weight $k$ if

(weight $k$ - Jac) $\quad\left(\begin{array}{cc}A & 0 \\ 0 & -{ }^{\top} A\end{array}\right) v=k \operatorname{Tr} A v \quad(\forall A \in \mathfrak{g l}(n, \mathbb{C}))$.

Suppose that $v_{0}$ is holomorphic of index $\mathcal{M}$ and weight $\left(-r+\frac{n+j+1}{2}\right)$. Then $\hat{M}_{+}^{r} v_{0}$ is holomorphic of index $\mathcal{M}$ and weight $\left(r+\frac{n+j+1}{2}\right)$. Moreover, if $k \neq r$ then $\hat{M}_{+}^{k} v_{0}$ is of weight $\left(-r+\frac{n+j+1}{2}+2 k\right)$ and index $\mathcal{M}$, but not holomorphic.

\section{Proof of Theorem 8.7}

Definition 9.1. Let $\mathfrak{v}$ denote the subspace of $\mathfrak{h}^{(n, j)}$ consisting of all matrices of the form

$$
\left(\begin{array}{cccc}
0 & 0 & 0 & { }^{\top} u \\
v & 0 & u & 0 \\
0 & 0 & 0 & -{ }^{\top} v \\
0 & 0 & 0 & 0
\end{array}\right),
$$

and let $\mathfrak{z}$ denote the center of $\mathfrak{g}^{(n, j)}$. 
Definition 9.2. Define $T_{0}: \mathfrak{h}^{(n, j)} \rightarrow \mathfrak{s p}(2 n)$ by

$$
\begin{aligned}
T_{0}\left(\begin{array}{cccc}
0 & 0 & 0 & { }^{\top} u \\
v & 0 & u & Z^{\top} \\
0 & 0 & 0 & -{ }^{\top} v \\
0 & 0 & 0 & 0
\end{array}\right) & =\left(\begin{array}{cccc}
0 & 0 & 0 & { }^{\top} u \\
v & 0 & u & 0 \\
0 & 0 & 0 & -{ }^{\top} v \\
0 & 0 & 0 & 0
\end{array}\right)\left(\begin{array}{cccc}
0 & 0 & 0 & 0 \\
0 & 0 & 0 & 0 \\
0 & 0 & 0 & 0 \\
0 & \widetilde{Z} & 0 & 0
\end{array}\right)\left(\begin{array}{cccc}
0 & 0 & 0 & { }^{\top} u \\
v & 0 & u & 0 \\
0 & 0 & 0 & -{ }^{\top} v \\
0 & 0 & 0 & 0
\end{array}\right) \\
& =\left(\begin{array}{cccc}
{ }^{\top} u \widetilde{Z} v & 0 & { }^{\top} u \widetilde{Z} u & 0 \\
0 & 0 & 0 & 0 \\
-{ }^{\top} v \widetilde{Z} v & 0 & -{ }^{\top} v \widetilde{Z} u & 0 \\
0 & 0 & 0 & 0
\end{array}\right),
\end{aligned}
$$

where $\widetilde{Z}$ is the "classical adjoint" of $Z$.

Observe that $T_{0}$ is $\mathfrak{s p}(2 n)$-equivariant. Furthermore, it is nonzero. As the adjoint representation of $\mathfrak{s p}(2 n)$ is irreducible, it follows that $T_{0}$ is surjective. This gives an injective, $\mathfrak{s p}(2 n)$-equivariant map from the dual of $\mathfrak{s p}(2 n)$ into the space of polynomial functions on $\mathfrak{h}^{(n, j)}$. One may identify $\mathfrak{g}^{(n, j)}$ with its dual using the invariant bilinear form $(X, Y) \mapsto \operatorname{Tr}(X Y)$. Then each of the spaces $\mathfrak{s p}(2 n), \mathfrak{v}$ and $\mathfrak{z}$ is identified with its dual and we obtain an injective $\mathfrak{s p}(2 n)$-equivariant map $T: \mathfrak{s p}(2 n) \rightarrow \operatorname{sym}^{2} \mathfrak{v} \otimes \operatorname{sym}^{j-1} \mathfrak{z}$.

The map $T$ may be described concretely as follows. Define $\underline{Z} \in \operatorname{sym}_{j}^{2}(\mathfrak{z})$ to be the $j \times j$ matrix with $i, l$ entry equal to $\left(E_{n+i, 2 n+j+l}+E_{n+l, 2 n+j+i}\right) \in \mathfrak{z}$, define $\underline{\mathrm{V}} \in \operatorname{Mat}_{j \times n} \mathfrak{v}$ to be the $j \times n$ matrix with $i, l$ entry equal to $E_{n+i, l}-$ $E_{n+j+l, 2 n+j+i} \in \mathfrak{v}$, and define $\underline{\mathrm{U}} \in \mathrm{Mat}_{j \times n} \mathfrak{v}$ to be the $j \times n$ matrix with $i, l$ entry equal to $E_{n+i, n+j+l}+E_{l, 2 n+j+i} \in \mathfrak{v}$. Then

$$
T(X)=\operatorname{Tr}\left({ }^{\top} X\left[\begin{array}{c}
{ }^{\top} \underline{\mathrm{U}} \\
{ }^{\top} \underline{\mathrm{V}}
\end{array}\right] \underline{\widetilde{Z}}\left[\begin{array}{ll}
\underline{\mathrm{V}} & \underline{\mathrm{U}}
\end{array}\right]\right) .
$$

Proposition 9.3. For all $V \in \mathfrak{v}$ and all $X \in \mathfrak{s p}(2 n)$ one has

$$
\operatorname{ad}(V) T(X)=2 \operatorname{det} \underline{Z} \cdot[V, X] .
$$

Proof. The space

$$
\{X \in \mathfrak{s p}(2 n): 2 \operatorname{det} \underline{\mathrm{Z}} \cdot[V, X]-\operatorname{ad}(V) \cdot T(X)=0 \forall V \in \mathfrak{v}\}
$$

is clearly an $\mathfrak{s p}(2 n)$-submodule. We claim that it is $\mathfrak{s p}(2 n)$. It suffices to show that it is nonzero. The space $\mathfrak{v}$ has a basis

$$
\begin{array}{r}
\left\{E_{n+i, l}-E_{n+j+l, 2 n+j+i}: 1 \leq i \leq j, 1 \leq l \leq n\right\} \\
\cup\left\{E_{n+i, n+j+l}+E_{l, 2 n+j+i}: 1 \leq i \leq j, 1 \leq l \leq n\right\} .
\end{array}
$$

It suffices to consider $V$ in this basis. We henceforth assume $V \in \mathfrak{v}$ is in this basis.

Take $X=E_{1, n+j+1}$. Then

$$
[V, X]= \begin{cases}E_{n+i, n+j+1}+E_{1,2 n+j+i} & V=E_{n+i, 1}-E_{n+j+1,2 n+j+i}(1 \leq i \leq j) \\ 0, & V \text { not of this form. }\end{cases}
$$


One may express $T(X)$, as $\underline{\underline{u}} \underline{\widetilde{Z}}^{\top} \underline{\mathrm{u}}$, where

$$
\underline{\mathrm{u}}=\left[E_{n+1, n+j+1}+E_{1,2 n+j+1}, \ldots, E_{n+j, n+j+1}+E_{1,2 n+2 j}\right] \in \operatorname{Mat}_{1 \times j} \mathfrak{v},
$$

and the product should be computed in the symmetric algebra of $\mathfrak{h}^{(n, j)}$. Now, $V$ acts trivially on $\mathfrak{z}$, so

$$
\operatorname{ad}(V)\left(\underline{u} \underline{\widetilde{Z}}^{\top} \underline{u}\right)=(\operatorname{ad}(V) \underline{u}) \underline{\widetilde{Z}}^{\top} \underline{u}+\underline{u} \widetilde{Z}\left(\operatorname{ad}(V)^{\top} \underline{u}\right) .
$$

Further,

$$
\operatorname{ad}(V) U=\left\{\begin{array}{l}
{\left[E_{n+1,2 n+j+i}+E_{n+i, 2 n+j+1}, \ldots, E_{n+j, 2 n+j+i}+E_{n+i, 2 n+2 j}\right],} \\
V=E_{n+i, 1}-E_{n+j+1,2 n+j+i}(1 \leq i \leq j) \\
0, V \text { not of this form. }
\end{array}\right.
$$

In the case when $\operatorname{ad}(V) \underline{u}$ is nonzero it agrees with the $i$ th row/column of the symmetric matrix $\underline{Z}$. It follows that $\operatorname{ad}(V) \underline{u} \widetilde{Z}$ is equal to $\operatorname{det} \underline{Z}$ times the $i$ th standard basis vector. Hence, $(\operatorname{ad}(V) \underline{\mathrm{u}}) \underline{\widetilde{Z}}^{\top} \underline{\underline{u}}$ equals $\operatorname{det} \underline{\mathrm{Z}}$ times the $i$ th entry of $\underline{\mathrm{u}}$, which is $E_{n+i, n+j+1}+E_{1,2 n+j+i}$. It follows by symmetry that $\operatorname{ad}(V) T(X)=$ $2 \operatorname{det} \underline{\mathrm{Z}}\left(E_{n+i, n+j+1}+E_{1,2 n+j+i}\right)$.

Definition 9.4. Define $\underline{\mathrm{T}}: \mathfrak{s p}(2 n) \rightarrow U\left(\mathfrak{g}^{(n, j)}\right)$ by $\underline{\mathrm{T}}(X)=\lambda(2 \operatorname{det} \underline{\mathrm{Z}} \cdot X-$ $T(X))=2 \operatorname{det} \underline{\mathrm{Z}} \cdot X-\lambda(T(X))$. Here, $\lambda$ denotes symmetrization as on [6], p. 128 .

It follows from Proposition 9.3 that the image of $\underline{\mathrm{T}}$ commutes with $U\left(\mathfrak{h}^{(n, j)}\right)$ $\subset U\left(\mathfrak{g}^{(n, j)}\right)$.

Proposition 9.5. One has

$$
\underline{T}(X) \underline{T}(Y)-\underline{T}(Y) \underline{T}(X)=:[\underline{T}(X), \underline{T}(Y)]=2 \operatorname{det} \underline{Z} \underline{T}([X, Y]) .
$$

Proof. Indeed,

$$
\begin{aligned}
2 \operatorname{det} \underline{\mathrm{Z}}([X, Y]) & =4(\operatorname{det} \underline{\mathrm{Z}})^{2}[X, Y]-\operatorname{det} \underline{\mathrm{Z}} \lambda(\operatorname{ad}(X) T(Y)) \\
& =4(\operatorname{det} \underline{\mathrm{Z}})^{2}[X, Y]-2 \operatorname{det} \underline{\mathrm{Z}}[X, \lambda(T(Y))], \\
{[\underline{\mathrm{T}}(X), \underline{\mathrm{T}}(Y)] } & =[2 \operatorname{det} \underline{\mathrm{Z}} \cdot X-\lambda(T(X)), \underline{\mathrm{T}}(Y)]=2 \operatorname{det} \underline{\mathrm{Z}}[X, \underline{\mathrm{T}}(Y)] \\
& =4(\operatorname{det} \underline{\mathrm{Z}})^{2}[X, Y]-2 \operatorname{det} \underline{\mathrm{Z}}[X, \lambda(T(Y))] .
\end{aligned}
$$

Definition 9.6. Let $V$ be a $\mathfrak{g}^{(n, j)}$-module such that $[0,0, \zeta] \cdot v=2 \pi i \operatorname{Tr}(\mathcal{M} \zeta) v$ for all $\zeta \in \operatorname{Mat}_{j \times j}$, symmetric, and all $v \in V$. Define $X * v=\frac{1}{2 \operatorname{det} \mathcal{M}} \underline{\mathrm{T}}(X) v$. The previous proposition shows that $X * Y * v-Y * X * v=[X, Y] * v$. Hence, * extends to an action of $U(\mathfrak{s p}(2 n))$ on $V$.

Lemma 9.7. Let $V$ be a $U\left(\mathfrak{g}_{\mathbb{C}}^{(n, j)}\right)$-module with action $\cdot$ and define an alternate action of $\mathfrak{s p}(2 n, \mathbb{C})$ by $*$ as above. Suppose $v \in V$ satisfies

$$
\left(\begin{array}{ll}
0 & 0 \\
X & 0
\end{array}\right) \cdot v=[w, 0,0] \cdot v=0, \forall X \in \operatorname{Mat}_{n \times n} \mathbb{C}, \text { symmetric, } w \in \operatorname{Mat}_{j \times n} \mathbb{C} \text {. }
$$


Then

$$
\left(\begin{array}{cc}
0 & 0 \\
X & 0
\end{array}\right) * v=0 \forall X \in \text { Mat }_{n \times n} \mathbb{C}, \text { symmetric. }
$$

Proof. Write $\mathfrak{v}=\mathfrak{l}+\mathfrak{r}$ where $\mathfrak{l}=\left\{[w, 0,0], w \in \operatorname{Mat}_{j \times n}\right\}$ and $\mathfrak{r}=\{[0, \mu, 0], \mu \in$ $\left.\operatorname{Mat}_{j \times n}\right\}$. Then it follows from the definition of $T$ that

$$
T\left(\begin{array}{ll}
0 & 0 \\
X & 0
\end{array}\right) \in \operatorname{sym}^{2} \mathfrak{l} \subset \operatorname{sym}^{2} \mathfrak{v}
$$

and the result follows.

Lemma 9.8. Fix $\mathcal{M} \in \mathrm{Mat}_{j \times j} \mathbb{Z}$, symmetric. Let $V$ be a $U\left(\mathfrak{g}_{\mathbb{C}}^{(n, j)}\right)$-module such that $[0,0, \zeta] \cdot v=2 \pi i \operatorname{Tr}(\mathcal{M} \zeta) v$ for all $\zeta \in \operatorname{Mat}_{j \times j} \mathbb{C}$ and $v \in V$. Let . denote the given action of $\mathfrak{g}_{\mathbb{C}}^{(n, j)}$ on $V$ and define a second action $*$ of $\mathfrak{s p}(2 n, \mathbb{C})$ as in Definition 9.6. Suppose $v_{0} \in V$ satisfies

$$
[w, 0,0] \cdot v=0 \quad \forall w \in \operatorname{Mat}_{j \times n} \mathbb{C} .
$$

Then

$$
\lambda\left(T\left(\begin{array}{cc}
X & \\
& { }^{\top} X
\end{array}\right)\right) \cdot v=\frac{j}{2} \operatorname{Tr} X \cdot v
$$

whence

$$
\left(\begin{array}{cc}
X & \\
& { }^{\top} X
\end{array}\right) \cdot v=k \operatorname{Tr}(X) v \Longrightarrow\left(\begin{array}{cc}
X & \\
& { }^{\top} X
\end{array}\right) * v=\left(k-\frac{j}{2}\right) \operatorname{Tr}(X) v
$$

Proof. Write $V_{2 n}$ for the standard representation of $S p(2 n)$, realized as column vectors, and write $U_{j}$ for the standard representation of $G L(j)$, also realized as column vectors. Keeping in mind that $\mathfrak{g}^{(n, j)}$ inherits an action of $S p(2 n) \times$ $G L(j)$ from inclusion into $\mathfrak{s p}(2 n+2 j)$, one has isomorphisms

$$
\begin{aligned}
\mathfrak{s p}(2 n) & \cong \operatorname{sym}^{2} V_{2 n}, & v_{1} \cdot v_{2} \in \operatorname{sym}^{2} V_{2 n} \mapsto\left(v_{1}{ }^{\top} v_{2}+v_{2}{ }^{\top} v_{1}\right) J \in \mathfrak{s p}(2 n), \\
\mathfrak{v} & \cong U_{j} \otimes V_{2 n}, & v \otimes u \in U_{j} \otimes V_{2 n} \mapsto\left[u^{\top} v, 0\right] \in \mathfrak{v}, \\
\mathfrak{z} & \cong \operatorname{sym}^{2} U_{j}, & u_{1} \cdot u_{2} \in \operatorname{sym}^{2} U_{j} \mapsto u_{1}{ }^{\top} u_{2}+u_{2}{ }^{\top} u_{1}
\end{aligned}
$$

(Here, we identify the $j \times 2 n$ matrix $u^{\top} v$ with a pair of $j \times n$ matrices $\nu, \mu$ to obtain an element of $\mathfrak{v}$ in the usual form).

The vector space $V_{2 n}$ is the direct sum of two $n$-dimensional isotropic subspaces $W, W^{\perp}$ such that $\mathfrak{l} \leftrightarrow W \otimes U_{j}$ and $\mathfrak{r} \leftrightarrow W^{\perp} \otimes U_{j}$. Specifically, $W$ is the span of the first $n$ standard basis vectors and $W^{\perp}$ is the span of he last $n$.

Hence $v_{1} \cdot v_{2} \in \operatorname{sym}^{2} V_{2 n}$ corresponds to a matrix of the form

$$
\left(\begin{array}{cc}
X & 0 \\
0 & -{ }^{\top} X
\end{array}\right)
$$

if and only if one of $v_{1}, v_{2}$ lies in $W$ and the other in $W^{\perp}$. Without loss of generality one may assume $v_{1} \in W$ and $v_{2} \in W^{\perp}$. 
Now, let $u_{1}, \ldots, u_{j}$ denote the standard basis basis for $U_{j}$. Then the mapping $T$ can be expressed in this notation as

$$
T\left(v_{1} \cdot v_{2}\right)=\sum_{i_{1}, i_{2}=1}^{j}\left(v_{1} \otimes u_{i_{1}}\right) \widetilde{\mathbf{Z}}_{i_{1}, i_{2}}\left(v_{2} \otimes u_{i_{2}}\right) .
$$

Furthermore,

$$
[0,0, \zeta] \cdot v=2 \pi i \operatorname{Tr}(\mathcal{M} \zeta) v \Longrightarrow \underline{\mathrm{Z}}_{i j} v=4 \pi i \mathcal{M}_{i j} v
$$

Finally,

$$
\begin{aligned}
& \lambda\left(\left(v_{1} \otimes u_{i_{1}}\right) \widetilde{\mathbf{Z}}_{i_{1}, i_{2}}\left(v_{2} \otimes u_{i_{2}}\right)\right) \\
= & \frac{1}{2} \widetilde{Z}_{i_{1}, i_{2}}\left(\left(v_{1} \otimes u_{i_{1}}\right)\left(v_{2} \otimes u_{i_{2}}\right)+\left(v_{2} \otimes u_{i_{2}}\right)\left(v_{1} \otimes u_{i_{1}}\right)\right) \\
= & \frac{1}{2} \widetilde{Z}_{i_{1}, i_{2}}\left(\left[\left(v_{1} \otimes u_{i_{1}}\right),\left(v_{2} \otimes u_{i_{2}}\right)\right]+2\left(v_{2} \otimes u_{i_{2}}\right)\left(v_{1} \otimes u_{i_{1}}\right)\right) .
\end{aligned}
$$

Now, tracing through the definitions, $\left[\left(v_{1} \otimes u_{i_{1}}\right),\left(v_{2} \otimes u_{i_{2}}\right)\right]$ is precisely $\underline{\mathrm{Z}}_{i_{1}, i_{2}}$. Hence

$$
\sum_{i_{1}, i_{2}=1}^{j} \widetilde{\widetilde{Z}}_{i_{1}, i_{2}}\left[\left(v_{1} \otimes u_{i_{1}}\right),\left(v_{2} \otimes u_{i_{2}}\right)\right]=j(\operatorname{det} \underline{\mathrm{Z}}) .
$$

On the other hand, $v_{1} \in W \Longrightarrow v_{1} \otimes u_{i_{1}} \in \mathfrak{l} \Longrightarrow\left(v_{1} \otimes u_{i_{1}}\right) \cdot v=0$ for all $1 \leq i_{1} \leq j$. The result follows.

Definition 9.9. Fix a positive integer $N$. Let $\hat{\mathrm{M}}_{+, N}$ to be the $N \times N$ matrix whose $i, j$ entry is $E_{i, N+j}+E_{j, N+i} \in \mathfrak{s p}(2 N) \subset U(\mathfrak{s p}(2 n))$. Let $\hat{M}_{+, N}$ be the element of $U(\mathfrak{s p}(2 N))$ obtained by taking the determinant of this matrix.

One may note that the entries of $\hat{\mathrm{M}}_{+, N}$ all commute in $U(\mathfrak{s p}(2 N))$, so one does not need to be concerned about the orders in which the products are taken in taking the determinant. Furthermore, $\hat{M}_{+, n+j} \in U\left(\mathfrak{g}^{(n, j)}\right) \subset U(\mathfrak{s p}(2 n+2 j))$.

Lemma 9.10. One has $(\operatorname{det} \underline{Z})^{j} \underline{T}\left(\hat{M}_{+, n}\right)=(\operatorname{det} \underline{Z})^{n} \hat{M}_{+, n+j}$.

Proof. Let $\underline{\mathrm{U}}$ be the $j \times n$ matrix with $i, k$ entry equal to $\left[0, E_{i, k}, 0\right] \in U\left(\mathfrak{h}^{(n, j)}\right) \subset$ $U(\mathfrak{s p}(2 n+2 j))$. Then

$$
\underline{\hat{\mathrm{M}}}_{+, n+j}=\left(\begin{array}{cc}
\hat{\hat{\mathrm{M}}}_{+, n} & { }^{\top} \underline{\mathrm{U}} \\
\underline{\mathrm{U}} & \underline{\mathrm{Z}}
\end{array}\right) .
$$

The mapping $\underline{\mathrm{T}}: U(\mathfrak{s p}(2 n)) \rightarrow U\left(\mathfrak{g}^{(n, j)}\right)$ induces a mapping

$$
\operatorname{Mat}_{n \times n}(U(\mathfrak{s p}(2 n))) \rightarrow \operatorname{Mat}_{n \times n}\left(U\left(\mathfrak{g}^{(n, j)}\right)\right)
$$

which we denote by the same symbol $\underline{\mathrm{T}}$. Then $\underline{\mathrm{T}}\left(\hat{\mathrm{M}}_{+, n}\right)=\operatorname{det} \underline{\mathrm{Z}} \hat{\mathrm{M}}_{+, n}-\underline{\mathrm{U}}^{\top} \underline{\mathrm{U}}^{\top}$. Hence

$$
\operatorname{det} \underline{\mathrm{Z}}^{n} \cdot \hat{M}_{+, n+j}=\operatorname{det}\left(\begin{array}{cc}
\operatorname{det} \underline{\mathrm{Z}} \cdot I_{n} & -{ }^{\top} \underline{\mathrm{U}} \underline{\widetilde{Z}} \\
0 & I_{j}
\end{array}\right)\left(\begin{array}{cc}
\hat{\mathrm{M}}_{+, n} & { }^{\top} \underline{\mathrm{U}} \\
\underline{\mathrm{U}} & \underline{\mathrm{Z}}
\end{array}\right)
$$




$$
\begin{aligned}
& =\operatorname{det}\left(\begin{array}{cc}
\operatorname{det} \underline{\mathrm{Z}} \cdot \hat{\mathrm{M}}_{+, n}-{ }^{\top} \underline{\mathrm{U}} \underline{\widetilde{Z}} \underline{\mathrm{U}} & 0 \\
\underline{\mathrm{U}} & \operatorname{det} \underline{\mathrm{Z}} \cdot I_{j}
\end{array}\right) \\
& =(\operatorname{det} \underline{\mathrm{Z}})^{j} \operatorname{det} \underline{\mathrm{T}}\left(\underline{\hat{\mathrm{M}}}_{+, n}\right),
\end{aligned}
$$

which gives the result.

Proof of Theorem 8.7. Theorem 8.7 now follows from Proposition 2.1. Regard $V$ as a $U(\mathfrak{s p}(2 n, \mathbb{C}))$ module with the action $*$.

It follows from Lemma 9.7 that $v_{0}$ is holomorphic. By Lemma 9.8 it is of weight $-r+\frac{n+1}{2}$. Therefore, by Proposition 2.1, $\hat{M}_{+, n}^{r} * v_{0}$ is holomorphic and of weight $r+\frac{n+1}{2}$ relative to the action $*$. By Lemma 9.10, and the fact that $\mathcal{M}$ is assumed positive definite, the same is true of $\hat{M}_{+, n+j}^{r} \cdot v_{0}$. Lemma 9.8 shows that $\hat{M}_{+, n+j}^{r} \cdot v_{0}$ is of weight $r+\frac{n+j+1}{2}$ relative to the action $\cdot$ Now,

$$
\left(\begin{array}{cc}
X & 0 \\
0 & -{ }^{\top} X
\end{array}\right) \hat{M}_{+, n+j}-\hat{M}_{+, n+j}\left(\begin{array}{cc}
X & 0 \\
0 & -{ }^{\top} X
\end{array}\right)=\operatorname{Tr} X \hat{M}_{+, n+j}(\forall X \in \mathfrak{g l}(n+j, \mathbb{C})) .
$$

It immediately follows that

$$
\left(\begin{array}{cccc}
0 & 0 & 0 & 0 \\
\lambda & 0 & 0 & 0 \\
0 & 0 & 0 & -{ }^{\top} \lambda \\
0 & 0 & 0 & 0
\end{array}\right) \hat{M}_{+, n+j}^{k} \cdot v_{0}=0, \quad \forall \lambda \in \operatorname{Mat}_{j \times n} \mathbb{C}, k \in \mathbb{N} .
$$

It follows from this, along with Lemma 9.7 that $\hat{M}_{+, n+j}^{k} \cdot v_{0}$ is holomorphic if and only if $k=r$.

\section{References}

[1] R. Berndt and R. Schmidt, Elements of the Representation Theory of the Jacobi Group, Progress in Mathematics, 163. Birkhäuser Verlag, Basel, 1998.

[2] B. D. Boe, Homomorphisms between generalized Verma modules, Trans. Amer. Math. Soc. 288 (1985), no. 2, 791-799.

[3] _ Homomorphisms between generalized Verma modules, Ph.D. thesis, Yale University, 1982.

[4] G. Bol, Invarianten linearer differential gleichungen, Abh. Math. Sem. Univ. Hamburg 16 (1949), no. 3-4, 1-28.

[5] D. Bump, Automorphic Forms and Representations, Cambridge Studies in Advanced Mathematics, 55, Cambridge University Press, Cambridge, 1997.

[6] D. Bump and Y.-J. Choie, Derivatives of modular forms of negative weight, Pure Appl. Math. Q. 2 (2006), no. 1, part 1, 111-133.

[7] Y.-J. Choie and H. Kim, An analogy of Bol's result on Jacobi forms and Siegel modular forms, J. Math. Anal. Appl. 257 (2001), no. 1, 79-88.

[8] Harish-Chandra, The characters of semisimple Lie groups, Trans. Amer. Math. Soc. 83 (1956), 98-163.

[9] M. Harris, Special values of zeta functions attached to Siegel modular forms, Ann. Sci. École Norm. Sup. (4) 14 (1981), no. 1, 77-120. 
Department of Mathematics

SOUTHERN ILLINOIS UNIVERSITY

1245 LinCOLN DRIVE

MAilcode 4408

Carbondale, IL 62901, USA

E-mail address: jhundley@math.siu.edu 